\title{
SUPPLÉMENTATION PAR LA LYSINE ET LA MÉTHIONINE D'AlIMENTS CHEZ LE PORC EN CROISSANCE-FINITION. INFLUENGE DU SEXE ET DU RATIONNEMENT
}

\author{
J. LOUGNON \\ A. E. C. Société de Chimie organique et biologique, 03 -Commentry
}

\section{RÉSUMÉ}

L'influence du mode d'alimentation, du sexe, du taux d'amino-acides dans les régimes et les interactions entre ces facteurs sur les performances du Porc en croissance-finition sont étudiées au cours d'une expérience réalisée selon un schéma factoriel :

-Deux modes d'alimentation : ad libitum ou rationnée ;

- deux sexes : mâles castrés et femelles;

- deux types de régimes : carencés en lysine et méthionine par rapport aux besoins du porc rationné ou rééquilibrés par supplémentation.

La restriction alimentaire imposée par le rationnement se traduit par une baisse de la vitesse de croissance, une augmentation de l'indice de consommation et une diminution de l'épaisseur de lard des carcasses.

Les femelles consommant moins que les castrats ont une vitesse de croissance inférieure et sont plus maigres.

La supplémentation en amino-acides améliore la vitesse de croissance et l'efficacité alimentaire.

Le rationnement a une influence relative plus importante chez les castrats sur consommation journalière, vitesse de croissance et épaisseur du lard.

Les aliments supplémentés entraînent une diminution de l'adiposité des carcasses seulement dans le cas des porcs rationnés dont les besoins relatifs en amino-acides sont plus élevés.

Les effets favorables des taux plus élevés d'amino-acides sont beaucoup plus marqués chez les femelles.

\section{SUMMARY}

\section{IYSINE AND METHIONINE SUPPLEMENTED FEEDS FOR GROWING FINISHING PIGS EFFECTS OF SEX AND RATIONING}

The effect of feeding method, sex and amino acid ration content ant the interaction of these factors on growing-finishing pig performance are studied in a factorial experiment.

-2 feeding methods : ad libitum and restricted;

-2 sexes : castrated males and females; 
- 2 types of ration : lysine and methionine deficient in terms of restricted pig needs or rebalanced by supplementation.

Restricted feeding by rationing results in a lower growth rate, increase in feed conversion and a decrease of carcass backfat thickness.

- Females eat less than castrated males, have a lower growth rate and are leaner.

- Amino acid supplementation improves growth rate and feed efficiency.

- Rationing has a relatively greater effect on daily intake growth rate and backfat thickness in castrated males.

- Supplemented feeds cause a decrease of carcass adiposity only in restricted pigs with higher relative amino acid needs.

- The favourable effects of higher amino acid content are more marked in females.

\title{
UTILISATION DU TOURTEAU DE TOURNESOL PAR LE PORC EN CROISSANCE-FINITION
}

\author{
Frère E. RENOUX \\ Centre de Techniciens de l'Élevage, \\ Prieuré Notre-Dame-des-Bois, 27 - Canappeville
}

\section{RÉSUMÉ}

A la demande du CETIOM, nous avons cherché à comparer les incidences de la substitution du tourteau de tournesol au tourteau de soya supplémenté (ou non) en DL-méthionine, sur les performances des porcelets jusqu'à 90 jours, puis des porcs après go jours (GMQ, IC et qualités des carcasses). Les essais ont porté sur 3 séries de io portées soumises alternativement à chaque régime jusqu'à go jours et sur 3 échantillons de 13 mâles castrés issus de chaque série, après 90 jours La substitution du tournesol a été effectuée à raison de $6 \mathrm{p}$. roo de la ration. Le régime avec tournesol se caractérise par référence au régime soya par un meilleur rapport acides aminés soufrés/ lysine, mais une teneur en cellulose élevée. Dans le régime soya supplémenté en DL-méthionine, le rapport AA soufrés/lysine est rétabli, mais à un coût supérieur. On obtient une différence hautement significative favorable aux portées recevant du tournesol par rapport aux portées recevant le soya non supplémenté jusqu'à go jours. Aucune différence significative n'a été observée entre les autres régimes; or dans la conjoncture actuelle, l'utilisation de tournesol diminue le coût de l'aliment. 